\title{
A FAMILY OF NEWTON TYPE ITERATIVE PROCESSES .
}

\author{
M. A. Hernández \& M. A. Salanova \\ Universidad de La Rioja . Dpto. de Matemáticas . \\ Edificio de Magisterio . C/ Luis de Ulloa $s / n$. \\ 26001 . LOGROÑO . SPAIN .
}

\begin{abstract}
.
In this paper, we define an index of the convexity measure by means of the logarithmic operator. As an application, we study the influence of the convexity on Newton method for solving nonlinear equations. Then we define a family of Newton type iterative processes to solve a complex or scalar nonlinear equation, we can always apply an iterative process of this family faster than Newton method.
\end{abstract}

Classification A.M.S. 1985 . : 65 H 05,26 A 51

C.R. CATEGORIES : $5.1,5.15$

Keywords : Nonlinear equations. Iterative processes . Optimization . Convexity .

\section{Introduction.}

We begin this paper defining an index of the convexity measure for a function $h$ at each point. We prove that it is a good measure of the convexity and we make a comparative study of this one and the curvature of $h$. 
To continue we do an application of this concept. So we are going to prove that when we apply Newton method to solve a nonlinear equation

$$
h(x)=0
$$

the convexity of the function $h$ decides the speed of convergence of this iterative process. From this result we obtain the family of Newton type iterative processes given by the expression

$$
x_{n}^{\alpha}=H_{\alpha}\left(x_{n-1}^{\alpha}\right)=x_{n-1}^{\alpha}-\frac{h\left(x_{n-1}^{\alpha}\right)}{h\left(x_{n-1}^{\alpha}\right)}\left(1+\alpha h\left(x_{n-1}^{\alpha}\right)\right) \text { with } \alpha \geq 0 .
$$

Notice that all these iterative processes have the same order of convergence and efficiency index [7] than Newton method but they have more speed of convergence than this one. You can see that for $\alpha=0$ we obtain Newton method.

It is interesting to note that in the practice given (1) we compute the convexity of $h$, which allows us to know the appropriate value of $\alpha$ to obtain the fastest iterative process of the family (2) for solving this equation. The above optimization results assures us that this method converges to the root of (1) faster than Newton one.

To continue, we consider the parameter $\alpha$ as a function , $\alpha(x)$, and we study when we obtain iterative processes with cubical convergence . Then, we define a family of iterative processes. From the result of optimization obtained for this family, we do a comparative study of the known Halley and Chebyshev methods [1] .

To finish, we study the convergence of this family (2) on the complex plane by means of majorizing sequences [8] .

\section{2.- An index of convexity measure.}


Let $h / C(2)(V)$ be a convex function and $V$ a neighbourhood of a suitable $x_{0} /[a, b]$. The curvature $K,[6]$, is a measure of the convexity of a function at each point .

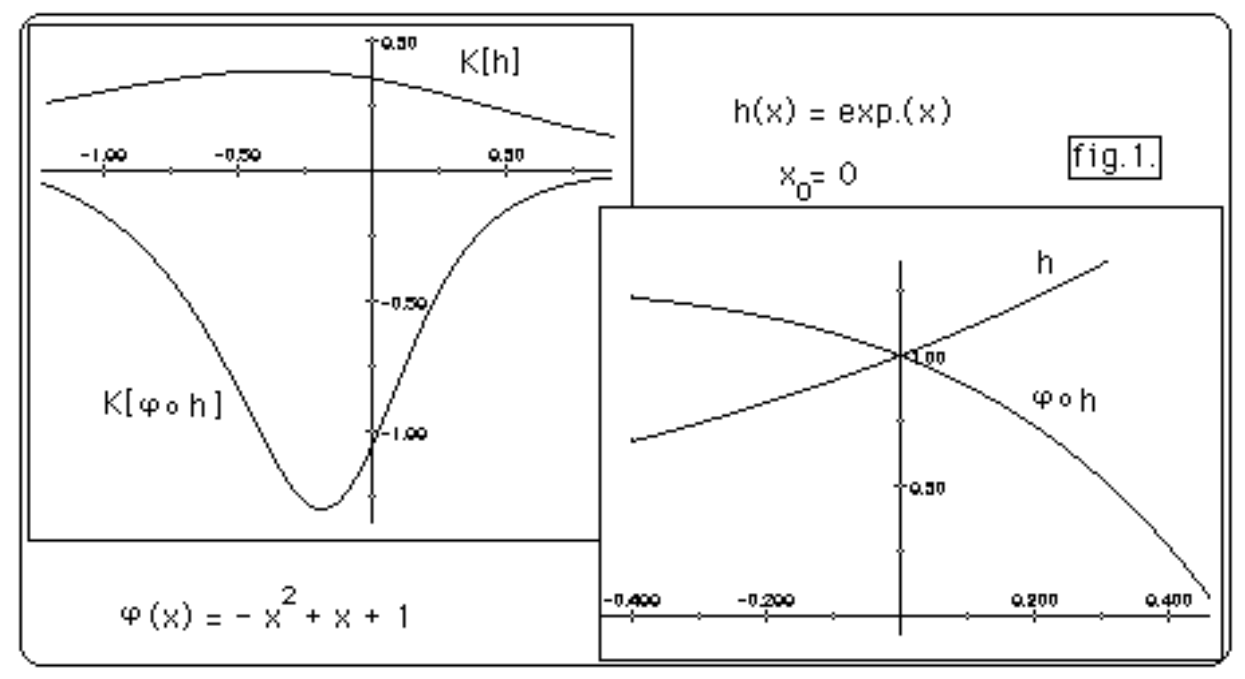

Notice that if $\|$ is a concave function in $C^{(2)}(U), h\left(x_{0}\right) / U$, with $\|\left(h\left(x_{0}\right)\right)=1$, it is clear that $\| \circ h$ has smaller curvature than

$h$, fig.1., since that $K(\varphi h)\left(x_{0}\right)=K(h)\left(x_{0}\right)+\frac{\varphi^{\prime \prime}\left(h\left(x_{0}\right)\right) h\left(x_{0}\right)^{2}}{\left[1+h\left(x_{0}\right)^{2}\right]^{3 / 2}}$. Hence, 
by applying a concave operator to a convex function we obtain a function with smaller curvature than the original one. For this, if we consider the logarithmic function and the convex function $T[h]$, with $T[h](x)=h(x)$ $h\left(x_{0}\right)+1$, we are in the previous conditions. Now, if we apply successively the logarithmic operator to $T[h]$ until we obtain a concave function, we can define an index of the convexity measure of a function at each point , called log-degree of convexity of $h$, considering the number of times that we need to apply the logarithmic operator to get a concave function . So, if we define $H_{n}(x)=\log G_{n-1}(x)$ with $G_{0}(x)=T[h](x)$ and $G_{n}(x)$ $=T\left[H_{n}\right](x)$ for $n \geq 1$, the sequence $\left\{H_{n}(x)\right\}$ will characterize the logdegree of convexity of $h$, since $K(h)\left(x_{0}\right)>K\left(H_{1}\right)\left(x_{0}\right)>\ldots>K\left(H_{n}\right)\left(x_{0}\right) \geq$ $0>K\left(H_{n+1}\right)\left(x_{0}\right)>\ldots$. ( fig.2. with $\left.x_{0}=0\right)$.

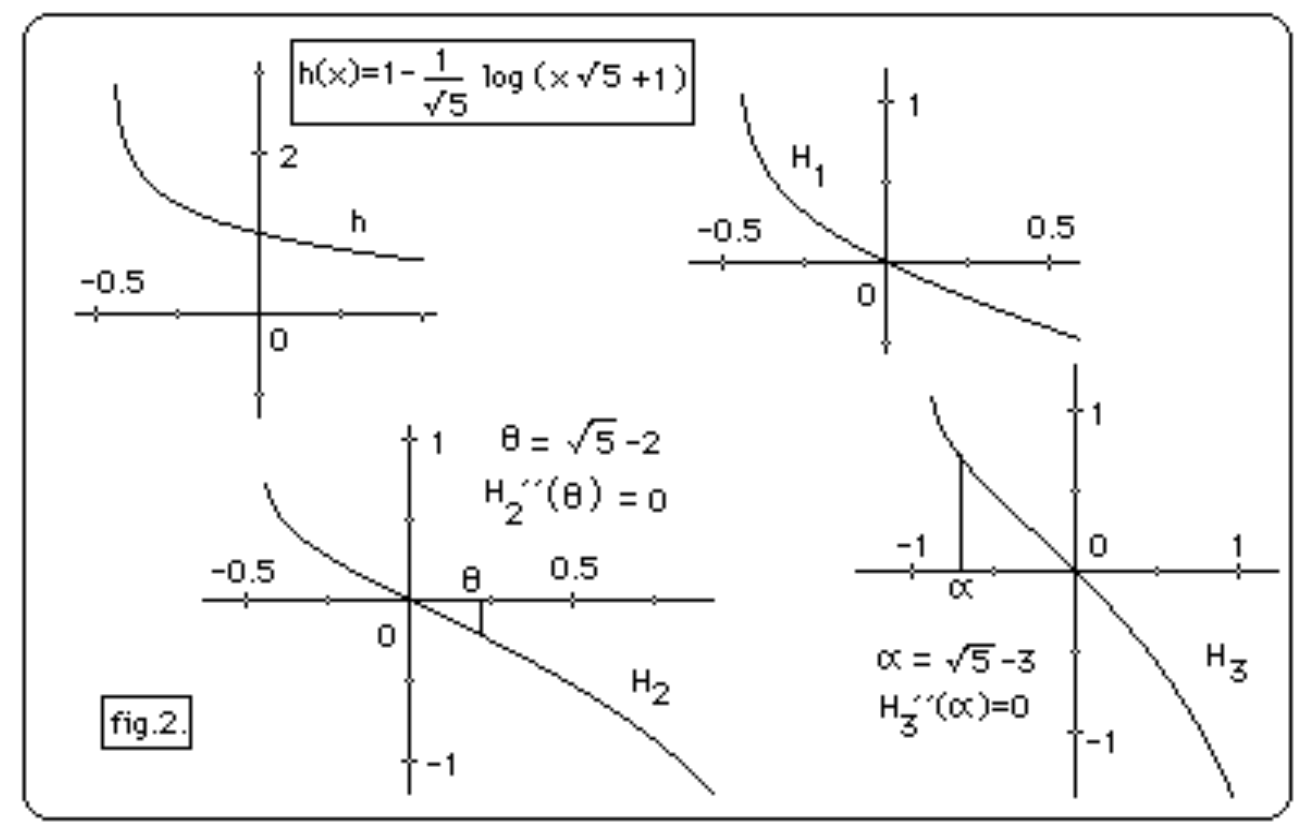

On the other hand, it is easy to prove, by induction, that $\mathrm{H}_{\mathrm{n}}{ }^{\prime \prime}\left(\mathrm{x}_{0}\right)$ $=h^{\prime \prime}\left(x_{0}\right)-n h^{\prime}\left(x_{0}\right)^{2}$ for all $n$, and therefore it follows that $H_{n}$ is convex at $x_{0}$ if and only if $h^{\prime \prime}\left(x_{0}\right)\left[h^{\prime}\left(x_{0}\right)\right]^{-2} \geq n$. When $x_{0}$ is a minimum of $f$, it follows that $H_{n}$ is convex at $x_{0}$ for all $n$, and 
conversely. Then, the log-degree of convexity of $h$ at $x_{0}$ is defined to be the positive real number given by $U[h]\left(x_{0}\right)=h^{\prime \prime}\left(x_{0}\right)\left[h^{\prime}\left(x_{0}\right)\right]^{-2}$. If $x_{0}$ is a minimum of $\mathrm{h}$ we set $\mathrm{U}[\mathrm{h}]\left(\mathrm{x}_{0}\right)=+\infty$.

Notice that the curvature and the log-degree of convexity of a function have a similar behaviour, except in a neighbourhood of the critic points. This situation does not exist in our conditions .

On the other hand, Roberts [9] points out some of the "good properties" that a measure of the convexity have to have. It is easy to prove that $U[h](x)$ satisfies some of these ones:

(i) $U[h]\left(x_{0}\right) \geq 0$ and $U[h](x)=0$ in $V$ if and only if $h$ is affine .

(ii) In our conditions, i.e., $h_{1}$ and $h_{2}$ increasing functions, it is verified that $U\left[h_{1}+h_{2}\right]\left(x_{0}\right) \leq U\left[h_{1}\right]\left(x_{0}\right)+U\left[h_{2}\right]\left(x_{0}\right)$.

\section{3.- Newton method and the convexity.}

As the functions $h$ and $h=h / h\left(x_{0}\right)$ have the same convexity respect to the logarithmic operator in the point $x_{0}$ and $h\left(x_{0}\right)=1$, if we apply the last study to the function $\underline{h}$ we obtain another convexity measure, the degree of logarithmic convexity [2], which

denote by $L_{h}\left(x_{0}\right)=U[h]=\frac{h\left(x_{0}\right) h^{\prime}\left(x_{0}\right)}{h^{\prime}\left(x_{0}\right)^{2}}$.

It is known [7], that if $h \in C^{(p)}([a, b]), p \geq 2$, verifies Fourier's conditions, i.e. $h(a) h(b)<0, h^{\prime} \neq 0$, the sign of $h^{\prime \prime}$ not changing in $[a, b]$, and besides we take $x_{0} \in[a, b]$ such that $h\left(x_{0}\right) h^{\prime \prime}\left(x_{0}\right)>0$, then the sequence $\left\{x_{n}\right\}$ given by 


$$
x_{n}=H\left(x_{n-1}\right)=x_{n-1}-\frac{h\left(x_{n-1}\right)}{h\left(x_{n-1}\right)}
$$

converges to the only root $s$ of $(1)$ in $[a, b]$. Besides this procedure has at least quadratic convergence .

From now on, we consider $h \in \mathrm{C}^{(\mathrm{p})}([\mathrm{a}, \mathrm{b}]), \mathrm{p} \geq 2, \mathrm{~h}(\mathrm{a}) \mathrm{h}(\mathrm{b})<0$, $\mathrm{h}^{\prime}>0$ and $\mathrm{h}^{\prime \prime} \geq 0$ in $[\mathrm{a}, \mathrm{b}]$.

By the geometric construction of Newton method it is clear that the convexity of $h$ influences in the convergence of the sequence $\left\{x_{n}\right\}$, as we can see in the following result. For this, we consider a function $g$ in the same conditions as the function $h$ and such that $g(x)=0$ is an equivalent equation to (1) in $[a, b]$, i.e., it has only the root $s$ in $[a, b]$. We denote $y_{n}=G\left(y_{n-1}\right)=y_{n-1}-\frac{g\left(y_{n-1}\right)}{g^{\prime}\left(y_{n-1}\right)}$ with $\mathrm{y}_{0}=\mathrm{x}_{0}$.

On these hypothesis the Newton method converges to the only root $s$ of (1) in $[a, b]$ for each $x_{0} \in[a, b]$ if $h\left(x_{0}\right)>0$ or if $h\left(x_{0}\right)<0$ when $b-x_{0} \geq-h\left(x_{0}\right) / h^{\prime}\left(x_{0}\right)$. From now, we assume this condition.

\section{Theorem 1.}

If $\left|L_{h}(x)\right|>\left|L_{g}(x)\right|$ in $[a, b]-\{s\}$, then the sequence $\left\{y_{n}\right\}$ converges to $s$ faster than $\left\{x_{n}\right\}$.

Proof: 
In our conditions, if $\mathrm{h}\left(\mathrm{x}_{0}\right)>0$ it is known [7] that $\left\{\mathrm{x}_{\mathrm{n}}\right\}$ and $\left\{\mathrm{y}_{\mathrm{n}}\right\}$ are decreasing sequences. Then, it is enough to prove that $y_{n}<x_{n}$ for all $n \in)$.

Applying the Mean Value Theorem, as $H$ and $G$ are increasing functions, we obtain $x_{k}-y_{k} \geq(H-G)\left(x_{k-1}\right)=\left(L_{h}\left(\theta_{k-1}\right)-L_{g}\left(\theta_{k-1}\right)\right)$ $\left(\mathrm{x}_{\mathrm{k}-1}-\mathrm{s}\right)>0$, for $\theta_{\mathrm{k}-1} \in\left(\mathrm{s}, \mathrm{x}_{\mathrm{k}-1}\right)$, and by an induction procedure it follows the thesis.

If $h\left(x_{0}\right)<0$, as $s=H(s) \leq x_{1}=H\left(x_{0}\right) \leq b$ it follows that $x_{n} \geq s$ for all $n \geq 1$, then the sequence $\left\{x_{n}\right\}$ decreases to $s$ for $n \geq 1$. On the other hand, as $y_{1}-x_{1}=G\left(x_{0}\right)-H\left(x_{0}\right)=\left(L_{g}-L_{h}\right)\left(\theta_{0}\right)\left(x_{0}-s\right)<0$ for $\theta_{0} \in\left(x_{0}, s\right)$. So , it is easy to prove thet $\left\{y_{n}\right\}$ decreases to $s$ for $n \geq 1$.

Therefore, as in the above case, it follows that $\left\{y_{n}\right\}$ converges to $s$ faster than $\left\{x_{n}\right\} . \quad \#$

Notice that it is enough that $g$ verify the last conditions in $\left(s, x_{0}\right]$ if $x_{0}>s$ or in $\left[x_{0}, x_{1}\right]$ if $x_{0}<s$.

From the last theorem, given the equation (1) we take the function $g(x)=\frac{h(x)}{1+\alpha h(x)}$ with $\alpha \geq 0$ and $1+\alpha h(x)>0$. It is cle

equation equivalent to (1). Then applying the above theorem we obtain conditions for each $\alpha$ such that the iterative process of the family (2) , converges to $\mathrm{s}$.

From now we denote by : 
$\langle p, q\rangle=[\min \cdot\{p, q\}, \max \cdot\{p, q\}]$

and for any interval $[\mathrm{p}, \mathrm{q}]$

Besides we assume $\left|L_{h}(x)\right|<2$ in $[a, b]$. Notice that this condition is not restrictive because $L_{h}(s)=0$.

\section{Theorem 2.}

decreasing and converges to $s$.

Moreover, if $0 \leq \alpha_{i} \leq \mathrm{m}\left\langle\mathrm{a}, \mathrm{b}, \mathrm{x}_{0}\right\rangle$ for $\mathrm{i}=1,2$ and $\alpha_{1} \leq \alpha_{2}$, then the iterative process corresponding to $\alpha_{2}$ converges to $s$ faster than the corresponding to $\alpha_{1}$.

Proof :

Notice that if $\alpha \leq m\left\langle a, b, x_{0}\right\rangle$ then $1+\alpha h(x)>0$ in $[a, b]$. On the

it is clear that $g^{\prime}(x)>0$ and $g^{\prime \prime}(x)>0$ in $[a, b]$, so the function $g$ verifies the same conditions as the function $h$ and thus $g(x)=0$ is an equation equivalent to (1).Besides, when $h\left(x_{0}\right)<0$ we have that 
$x_{1}^{\alpha}=x_{0}^{\alpha}-\frac{g\left(x_{0}^{\alpha}\right)}{g^{\prime}\left(x_{0}^{\alpha}\right)}=x_{0}^{\alpha}-\frac{h\left(x_{0}^{\alpha}\right)}{h^{\prime}\left(x_{0}^{\alpha}\right)}-\alpha \frac{h\left(x_{0}^{\alpha}\right)^{2}}{h\left(x_{0}^{\alpha}\right)} \leq b \quad$ by the election of

In these conditions the Newton method converges and so the sequence given by (2) is decreasing to $s$.

To prove the second statement, given $0 \leq \alpha_{i} \leq m\left\langle a, b, x_{0}\right\rangle$ for easy to prove that $\left|\mathrm{L}_{g_{2}}(\mathrm{x})\right|<\left|\mathrm{L}_{g_{1}}(\mathrm{x})\right|$ in $[\mathrm{a}, \mathrm{b}]\{\mathrm{s}\}$, since that $L_{g}(x)=L_{h}(x)-\alpha h(x)\left[2-L_{h}(x)\right]$. Therefore, applying the Theorem 1 we obtain the optimization result of the thesis. \#

To continue, we are going to study the convergence of the iterative processes (2) in other way, i.e., without consider its relation with Newton method, thus we can get, for this family, some results of global convergence that complete the ones of the Theorem 2 .

\section{Theorem 3 .}

If $x_{0} \in[a, b],\left|L_{h}(x)\right|<1$ in $[a, b]$ and $0 \leq \alpha \leq \min \cdot\left\{\frac{1}{2 h(b)},-\frac{1}{3 h(a)}\right.$

then the sequence given by (2) converges to $s$.

Proof: 
As $\mathrm{H}_{\alpha}^{\prime}(\mathrm{x})=\mathrm{L}_{h}(\mathrm{x})-\alpha \mathrm{h}(\mathrm{x})\left[2-\mathrm{L}_{h}(\mathrm{x})\right]$ and taking into account the cases $\mathrm{x}<\mathrm{s}$ and $\mathrm{x}>\mathrm{s}$ we can prove that $-1<\mathrm{H}_{\alpha}^{\prime}(\mathrm{x})<1$ in $[\mathrm{a}, \mathrm{b}]$. Therefore, there is $\mathrm{N} \in \llbracket, 0<\mathrm{N}<1$ such that $\left|\mathrm{H}_{\alpha}^{\prime}(\mathrm{x})\right|<\mathrm{N}$.

inductive procedure it follows that $\left|x_{n}^{\alpha}-s\right|<N^{n}\left|x_{0}^{\alpha}-s\right|$ for al $\mathrm{n} \geq 1$. Therefore the sequence converges to $\mathrm{s}$. \#

For the next result we choose $x_{0} \in[a, b]$ with $h\left(x_{0}\right)<0$ and such

Theorem 4.

Let $\mathrm{x}_{0} \in[\mathrm{a}, \mathrm{b}]$ be with $\mathrm{h}\left(\mathrm{x}_{0}\right)<0$ and $\alpha \in\left[\mathrm{M}\left[\mathrm{x}_{0}, \mathrm{~b}\right],-1 / \mathrm{h}\left(\mathrm{x}_{0}\right)\right]$, then the sequence given by (2) is increasing to $s$.

Moreover, if $\alpha_{1}<\alpha_{2}$ with $\alpha_{j} \in\left[M\left[x_{0}, b\right],-1 / h\left(x_{0}\right)\right]$, then

Proof:

As $\alpha \geq M\left[x_{0}, b\right]$ it follows that $H_{\alpha}^{\prime}(x) \geq 0$ in $\left[x_{0}, s\right]$, then can prove that the sequence is bounded by $\mathrm{s}$.

On the other hand and inductive procedure allow us to obtain that 
and $1+\alpha h(x)>0$ in $\left[x_{0}, s\right]$. So this sequence converges and taking limits in (2) we obtain that the limit is the root $s$ of (1).

As the asymptotic error constant [4], $C_{\alpha i}$, for the iterative processes (2) are $C_{\alpha i}=\left|\frac{f^{\prime \prime}(s)}{2 f(s)}-\alpha_{i} f^{\prime \prime}(s)\right|$ and $\alpha_{i} \geq M\left[x_{0}, b\right]$ it is clear that $\mathrm{C}_{\alpha 1}<\mathrm{C}_{\alpha 2}$. So the thesis is proved. \#

Now we are going to realize a practical study of these results. For this, we consider the nonlinear equation $x^{m}-r=0$, for $m>1, r$, $x \in \llbracket$ with $r>0, x \geq 0$. Then, for $h(x)=x^{m}-r$, we obtain

Therefore, if we consider, for example, $m=15$, and $r=1993$, and applying the Theorem 2 for $[a, b]=[1,1993], \quad x_{0}=5$ and $\alpha=m(\langle 1,10,1993\rangle)$, we get the results of the Table 1 . Notice that the Table 1 and 2 show the error between the exact value and $x_{n}$ in the respective sequences. Moreover all the calculations were made with 9 


\begin{tabular}{|c|c|c|c|}
\hline iteration & Herton & $a x=m(<1,10,199$ & 35) \\
\hline 1 & 8,340538606 & 8,340538606 & \\
\hline 2 & 7,67387194 & 7,090538606 & \\
\hline 3 & 7,051649718 & 6,438332806 & \\
\hline 4 & 6470908977 & 5.878537131 & \\
\hline 5 & 5,928864285 & 5369665001 & \\
\hline 6 & 5,422994574 & 4,89898482 & \\
\hline 7 & 4,950830843 & 4,461071583 & \\
\hline 8 & 4,510144694 & 4,052809825 & \\
\hline 9 & 4,098837623 & 3,671916783 & \\
\hline $10:$ & 3,714951025 & 3,316466728 & \\
\hline 11 & 3,356656871 & 2,984729971 & \\
\hline 12 & 3,022249000 & 2,675114542 & \\
\hline 13 & 2,710135036 & 2,366142068 & \\
\hline 14 & 2,418628751 & 2,116435293 & \\
\hline 15 & 2,146943118 & 1,864709857 & \\
\hline $16:$ & 1,893183809 & 1.629767989 & \\
\hline 17 & 1,6563434 & 1,410493677 & \\
\hline 18 & 1.435296589 & 1,205850119 & Tab. 1 \\
\hline 19 & 1,226997364 & 1,014862196 & \\
\hline 20 & 1,036480663 & 0,836731418 & $x_{0}=10$ \\
\hline
\end{tabular}

On the other hand, if we consider the Newton method we get the results of Tab. 2 . Therefore for $\alpha=M(<1,1,1993>)$ the iterative process converges faster than the correspondent for $\alpha=0$.

Nevertheless, when we apply the Theorem 4 is when actually we can see the difference between the speed of convergence of any process of the family and Newton method. So if we take $x_{0}=1$ we get the results given on the Table 2 .

This shows us the big increment in the speed of convergence taking into account the simplicity of the equation considered. 


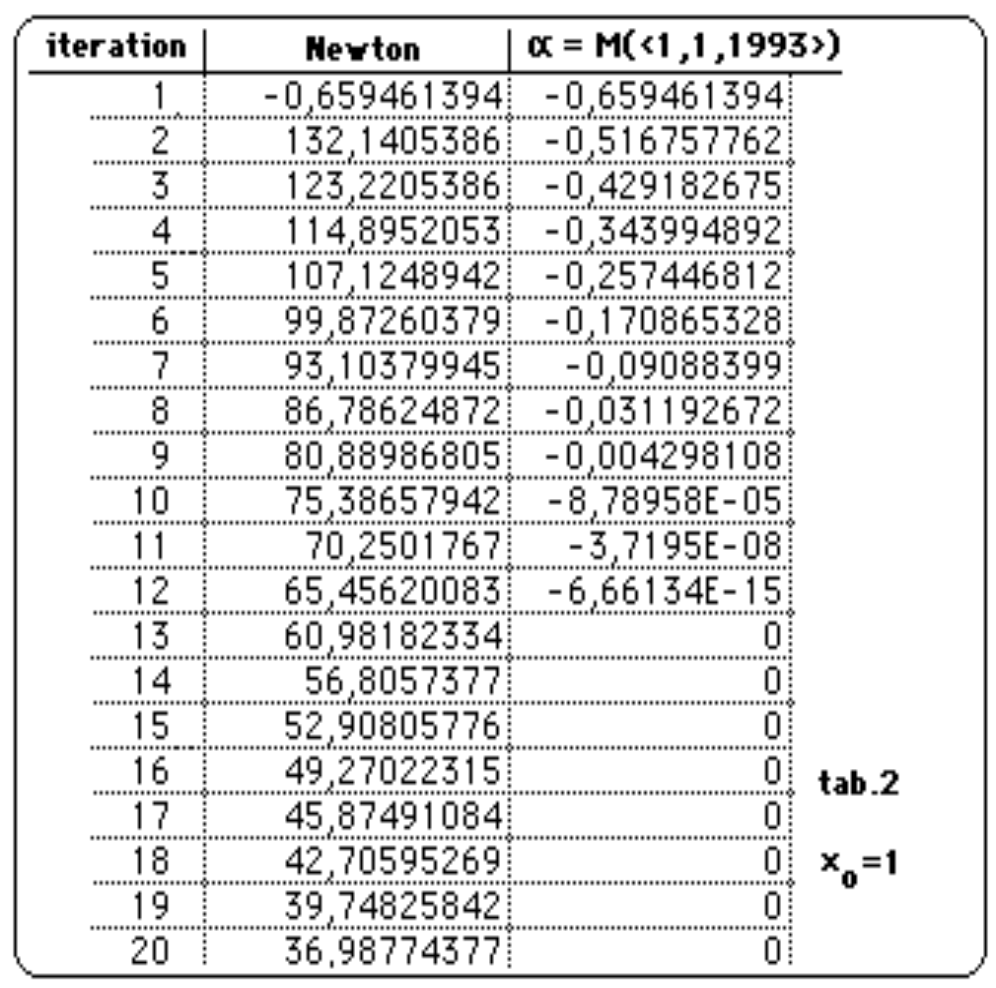

In the practice, given the equation (1) we compute $L_{h}$ and $U[h]$ and taking $\alpha=\mathrm{m}\left\langle\mathrm{a}, \mathrm{b}, \mathrm{x}_{0}\right\rangle$ or $\alpha=\mathrm{M}\left[\mathrm{x}_{0}, \mathrm{~b}\right]$, if $\mathrm{x}_{0} \in[\mathrm{a}, \mathrm{s}]$ we obtain the best iterative process of the family (2) to solve (1).

To finish, notice that all the results obtained in this Section are true with slight changes if $h$ is in the Fourier's conditions, since that, if $h$ verifies Fourier's conditions and we are not in the above hypothesis, we can always be in this situation changing $h(x)$ by $h(-x),-h(x)$ or $h(-x)$.

Besides, if we consider $\alpha \leq 0$ we can realize an analogous study of the iterative processes given by (2), but we are not interested in that at the moment .

To continue, we are going to study when we can obtain iterative processes with cubical convergence from this family . 
Now, if we consider $p \geq 4$, we can note that for $\alpha=U[h](s) / 2$ $\mathrm{H}_{\alpha}(\mathrm{s})=\mathrm{s}$ and $\mathrm{H}_{\alpha}^{\prime}(\mathrm{s})=\mathrm{H}^{\prime \prime}{ }_{\alpha}(\mathrm{s})=0$. So, it is known [1] that for this value of $\alpha$ we obtain an iterative process of third order. But as $s$ is unknown we cannot take this value of $\alpha$. Therefore if we consider $\alpha$ as a function, $\alpha(x)$, verif ying $0 \leq \frac{U[h](x)}{2} \leq \alpha(x) \leq \frac{U[h](x)}{2-L_{f}(x)}$, when $h(x) \geq 0$, and we take $H(x)=x-h(x)[1+\alpha(x) h(x)] / h^{\prime}(x)$, we obtain that all these iterative processes have cubical convergence .

Besides it is easy to note that for $\alpha=U[h](x) / 2$ and $\alpha=\mathrm{U}[\mathrm{h}](\mathrm{x}) /\left(2-\mathrm{L}_{\mathrm{h}}(\mathrm{x})\right)$ we obtain the Chebyshev and Halley methods respectively. So, this situation allow us to study the optimization for these functions $\alpha(x)$, and so to compare these last methods. For the optimization, when the iterative processes are decreasing, see [3] , we have that if $\alpha_{1}(x)<\alpha_{2}(x)$, and

we denote $x_{n}^{\alpha_{1}}=H_{\alpha_{1}}\left(x_{n-1}^{\alpha_{1}}\right)$ and $x_{n}^{\alpha_{2}}=H_{\alpha_{2}}\left(x_{n-1}^{\alpha_{2}}\right)$ with $x_{0}=x_{0}^{\alpha_{1}}=x_{0}^{\alpha_{2}}$ in $[a, b]$, then $x_{1}^{\alpha_{1}}-x_{1}^{\alpha}=\frac{h\left(x_{0}\right)^{2}}{h^{\prime}\left(x_{0}\right)}\left[\alpha_{2}\left(x_{0}\right)-\alpha_{1}\left(x_{0}\right)\right]>0$, and an inductive procedure assures us that $x_{n}{ }_{1}>x_{n}{ }^{\alpha}$ for each $n \in N$.

Therefore we can say that, in the decreasing case, the Halley method is better than the Chebyshev one.

\section{The family of Newton type iterative processes in the complex plane.}

In this Section we study the convergence of the family (2) to solve a nonlinear complex equation through the results obtained before. We 
assume that $D$ is an open and convex subset of $[$ and $f: D-->[$ an

holomorphic function in $D$. Given the nonlinear equation

(4)

$$
f(z)=0
$$

and the family of iterative processes

(5) $z_{n}^{\alpha}=F_{\alpha}\left(z_{n-1}^{\alpha}\right)=z_{n-1}^{\alpha}-\frac{f\left(z_{n-1}^{\alpha}\right)}{f\left(z_{n-1}^{\alpha}\right)}\left(1+\alpha f\left(z_{n-1}^{\alpha}\right)\right)$

we consider $z_{0} \in D$, denote $\eta=\left|\frac{f\left(z_{0}\right)}{f^{\prime}\left(z_{0}\right)}\right|$ and we make the real polinomial $p(x)=\frac{1}{4 \eta} x^{2}-2 x+2 \eta$.

To continue we will obtain some results of convergence and uniqueness for the family (5) by means of the Kantorovich type conditions [5] from the real nonlinear equation

(6)

$$
p(x)=0
$$

using mayorizing sequences [8]. We will denote $x^{*}=2 \eta(2-\sqrt{2})$ the smallest root of (6) .

\section{Theorem 5 .}

$$
\text { On the last conditions, let } \alpha \in\left[0, \frac{m}{16 \eta(m+1)}\right] \text { and } m \geq 1
$$

with $\mathrm{m} / \|$, verifying 
(i) $|f(z)|<\frac{p(x)}{m}$ when $\quad|z-z o| \leq x-x_{0}$

(ii ) $\left|U_{f}(z)\right| \leq \frac{m}{m+2 \alpha \eta}\left(\frac{m}{8 \eta}-2 \alpha(m+1)\right) \quad$ for $z \in \overline{B\left(z_{O}, x^{*}\right)}$

Then, for each $\alpha$ fixed, the iterative process given by (5) converges to the only root $z^{*}$ of (4) in $\overline{B\left(z_{0}, x^{*}\right)}$. Besides $\left|z-z_{0}\right| \leq x^{*}-x_{n}^{\alpha}$.

Proof :

Let $\left\{x_{n}^{\alpha}\right\}$ be the real sequence obtained aplying the family of iterative processes (2) for $\alpha \in\left[0, \frac{m}{16 \eta(m+1)}\right]$, $\mathrm{H}_{\alpha}=\mathrm{P}_{\alpha}, \mathrm{h}=\mathrm{p}$ and $\mathrm{x}_{0}=0$, notice that $\mathrm{p}$ verifies the Fourier's conditions in $\left[0, x^{*}\right]$.

In view of the comments following the Theorem 1 and as $U[p](x)=\frac{2 \eta}{(x-4 \eta)^{2}}$ is an increasing function and $\alpha<\frac{1}{16 \eta}=$ $\min .\left\{\frac{U[p](x)}{2} \mid x \in\left[0, x^{*}\right]\right\}<\min .\left\{\frac{U[p](x)}{2-L_{p}(x)} \mid x \in\left[0, x^{*}\right]\right\}$, it follows

$\left[0, x^{*}\right]$, being this sequence increasing to $x^{*}$.

Now we are going to prove that in ours hypotesis the sequence $\left\{x_{n}^{\alpha}\right\}$ mayorizes $\left\{z_{n}^{\alpha}\right\}$ and the results of the Theorem are obtained [5]. It is known [5] that the sequence $\left\{x_{n}^{\alpha}\right\}$ mayorizes $\left\{z_{n}^{\alpha}\right\}$ if the following conditions are verified

(a) $\left|F_{\alpha}\left(z_{0}\right)-z_{0}\right| \leq P_{\alpha}\left(x_{0}\right)-x_{0}$.

(b) $\left|F_{\alpha}^{\prime}(z)\right| \leq P_{\alpha}^{\prime}(x)$ when $\left|z-z_{0}\right| \leq x-x_{0}$.

The condition (a) is clear because $\left|F_{\alpha}\left(z_{0}\right)-z_{0}\right| \leq \eta(1+\alpha p(0) / m)$.

Taking into account that $p(x)$ is a decreasing function in $\left[0, x^{*}\right]$ and 
(i) , we obtain that $\left|F_{\alpha}^{\prime}(z)\right|=\left|L_{f}(z)+\alpha f(z) L_{f}(z)-2 \alpha f(z)\right|=$ $|f(z)|\left|U[f](z)+\alpha L_{f}(z)-2 \alpha\right| \underset{\left(^{*}\right)}{\leq} \frac{p(x)}{m}\left[|U[f](z)|\left(1+\frac{2 \alpha \eta}{m}\right)+2 \alpha\right]$. On the other hand as $U[p](x)(1+\alpha p(x))-2 \alpha \geq U[p](0)-2 \alpha=\frac{1}{8 \eta}-2 \alpha$ we have $P_{\alpha}^{\prime}(x) \underset{(* *)}{\geq} p(x)\left[\frac{1}{8 \eta}-2 \alpha\right]$. Then from $\left({ }^{*}\right),\left({ }^{*}\right)$ and (ii) it follows the condition (b). This completes the proof. \# Note: As $\frac{1}{2} \leq \frac{m}{m+1} \leq 1$ for $m \geq 1$, we can extend the values of $\alpha$ on $\prod_{+}$if we do a good election of the initial point $z_{0}$.

Now, we are going to do a study of the situations that can appear in the practice. On the first place, given $f(z)=0$ and $m \geq 1$ verifying the condition (i) from the Theorem 5 , we wonder if it always exist an iterative process of the family (5) to solve this equation. For that we take $z_{0} / D$ such that $M(U[f]) \leq 1 / 8 \eta$, where we denote

$$
M(U f])=\max \left\{\left\{|U[f](z)| \quad \mid z \in \overline{B\left(z_{0}, x^{*}\right)}\right\}\right.
$$

\section{Corollary 6.}

On the above conditions, given $m \geq 1$, such that $|f(z)| \leq p(x) / m$ when $\left|z-z_{0}\right| \leq x-x_{0}$, then there is $\alpha([0, m / 16 \eta(m+1))$ such that the

respective iterative processes of the family (5) converge to the only 


\section{Proof :}

We consider $S(m,-):[0,+\infty] \sqrt{ } \sqrt{ } \sqrt{ } \sqrt{ } \sqrt{ } \|$ with

$$
\text { (7) } S(m, \alpha)=\frac{m}{m+2 \alpha \eta}\left[\frac{m}{8 \eta}-2 \alpha(m+1)\right]
$$

As $M(U[\mathrm{f}]) \leq \mathrm{m} / 8 \eta=\mathrm{S}(\mathrm{m}, 0)$ and taking into account that $S\left(m, \frac{m}{16 \eta(m+1)}\right)=0$, it exists $\alpha \in\left[0, \frac{m}{16 \eta(m+1)}\right)$ such that $|\mathrm{U}[\mathrm{f}](\mathrm{z})| \leq \mathrm{S}(\mathrm{m}, \alpha)$. See ( fig. 3 ). Then, the Theorem 5. proves the result . \#

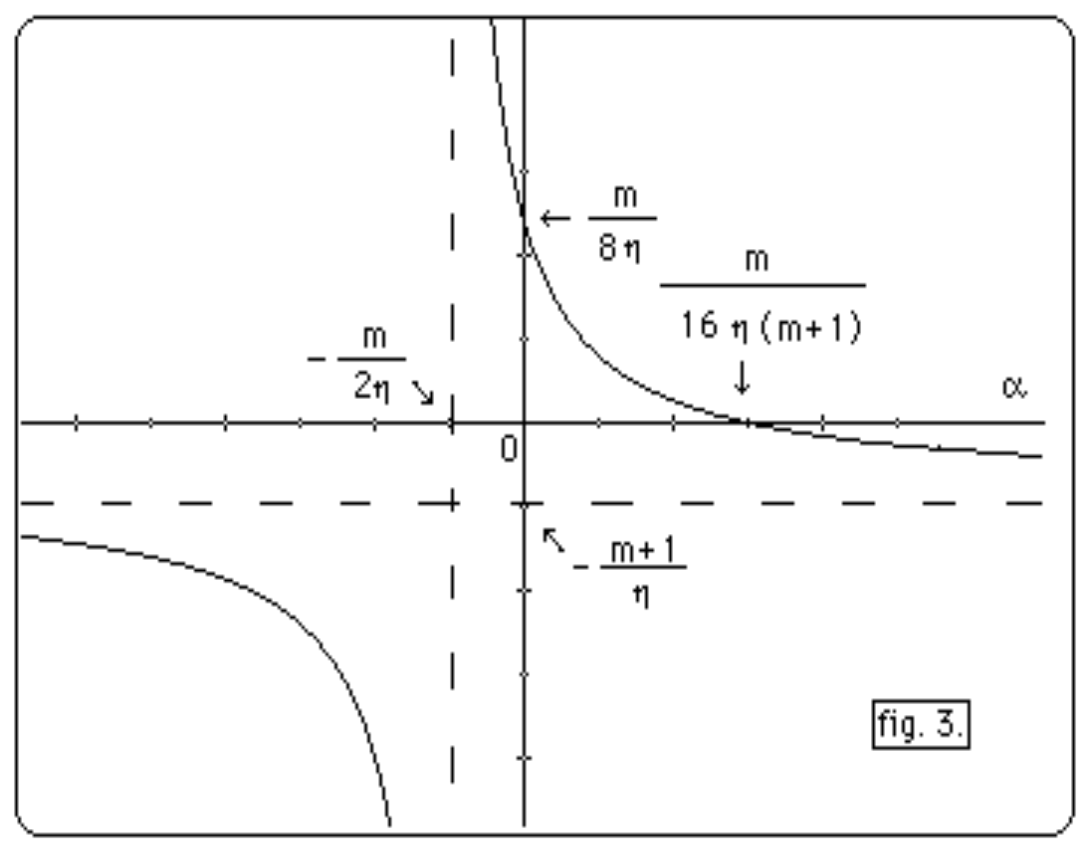

Notice that, by the optimization result obtained for the

$$
=M(U[f]) \text {. }
$$

To finish, we want to find convergence conditions for each iterative process of the family (5), i.e. for each value of $\alpha$ given in $[0,+\infty)$. Theorem 7. 
Given $\alpha /[0,+\infty)$, there exist $z_{0} \mid D$ and $m \geq 1$ such that $\alpha<\frac{m}{16 \eta(m+1)}$ and $|U[f](z)| \leq \frac{m}{m+2 \alpha \eta}\left[\frac{m}{8 \eta}-2 \alpha(m+1)\right]$ for $z \in \overline{B\left(z_{0}, x^{*}\right)}$.

Proof :

Given $\alpha([0,+\infty)$, as $1 / 2 \leq m /(m+1) \leq 1$, it is enough to take

$z_{0}\left\langle D\right.$ such that $\alpha<1 / 32 \eta$, being $\eta=\left|f\left(z_{0}\right) / f^{\prime}\left(z_{0}\right)\right|$. Moreover

On the other hand, we consider $S(-, \alpha):[1,+\infty) \sqrt{ } \sqrt{ }>\mathbb{}$, with

$S(m, \alpha)$ given by (7) .

It is clear that $S(1, \alpha)=\frac{1-32 \eta \alpha}{8 \eta(1+2 \eta \alpha)}$. Then it follows

that if $M(U[f]) \leq S(1, \alpha)$, we can take $m=1$. If $M(U[f])>S(1, \alpha)$, it exits $m_{0} / \|+$ such that $M(U[f])=S\left(m_{0}, \alpha\right)$. This proves the Theorem. 
From this result we can deduce that for each $\alpha /[0,+\infty)$ the

respective iterative process of (5) always converges if the condition of the Theorem 5 . is verified.

\section{REFERENCES}

[1] GANDER, W. : On Halley's iteration method . Am. Math .Monthly . 92 , oㅡ 2, (1985) , 131-134.

[2] HERNANDEZ, M.A. : A note on Halley's method . Numer. Math. 59, no 3 , (1991), 273-276 .

[3] HERNANDEZ, M. A. \& SALANOVA, M. A. : A family of Chebyshev-Halley type methods . Intern. J. Computer Math . 47 , № 1+2,(1993) , 59-63 .

[4] HOUSEHOLDER, A. S.: The numerical treatment of a single nonlinear equation .

Mc Graw Hill (1970) .

[5] KANTOROVICH, L. V. \& AKILOV, G. P. : Functional analysis . Pergamon Press (1982). 
[6] MILLMAN, R.S. \& PARKER, G.D.: Elements of differential geometry . Prentice Hall (1977) .

[7] OSTROWSKI, A.: Solution of equations in euclidean and banach spaces.

3th. Ed. Academic Press (1973) .

[8] RHEINBOLDT, W. C. : A unified convergence theory for a class of iterative process.

Siam J. Numer. Anal. 5 , (1968), 42-63.

[9] ROBERTS, A.W. \& VARBERG, D. E. : Convex functions . Academic Press . (1973) . 